\title{
Neumomediastino asociado al trabajo de parto: reporte de caso
}

Pneumomediastinum associated with labor: case report

\author{
Jhon Edison Ijají Piamba ${ }^{1^{*}}$ \\ Victoria Alejandra Rodríguez Castillo ${ }^{1}$ \\ Jorge Armando Cerón Bastidas ${ }^{1}$ \\ Sophía Hoyos Zúñiga ${ }^{2}$ \\ Jenny Cristina Gonzáles Dagua ${ }^{3}$ \\ Roberth Alirio Ortiz Martínez ${ }^{4}$
}

Recibido: 03 de febrero de 2017

Revisado: 19 de abril de 2017

Aceptado: 26 de mayo de 2017

\section{Resumen}

Antecedentes: El neumomediastino asociado al trabajo de parto es una entidad excepcional, con aproximadamente 200 casos reportados en todo el mundo. Resumen del caso clínico: Se presenta el caso de una mujer primigestante, quien en el postparto inmediato presenta disnea, dolor torácico, así como edemas en cara y cuello. Se realizó el diagnóstico de neumomediastino asociado al trabajo de parto; y se confirmó el mismo mediante radiografía y tomografía axial computarizada de tórax. La paciente recibió tratamiento conservador basado en medicación analgésica y aporte de oxígeno, con evolución clínica satisfactoria. Conclusiones: El diagnóstico del neumomediastino espontáneo es eminentemente clínico. Las medidas de soporte, incluyendo la oxigenoterapia, analgesia y ansiolisis, constituyen los pilares del tratamiento de esta patología.

Palabras clave: Enfisema mediastínico, enfisema subcutáneo, trabajo de parto, periodo posparto.

\begin{abstract}
Background: Pneumomediastinum associated with labor is an exceptional entity, with only around 200 cases reported worldwide. Clinical case summary: We present the case of a primigestante woman, who after a labor with prolonged expulsive period, presents clinical picture consisting of dyspnea, chest pain and edema of the face and neck. The diagnosis of pneumomediastinum associated with labor was made; and it was confirmed by radiography and computed tomography of the thorax. The patient received conservative treatment based on analgesic medication and oxygen supply with satisfactory clinical evolution. Conclusions: The diagnosis of spontaneous pneumomediastinum is eminently clinical. Support measures, including oxygen therapy, analgesia and anxiolysis, are the main basis of the treatment of this pathology.
\end{abstract}

Key words: Mediastinal emphysema, subcutaneous emphysema; labor, obstetric; postpartum period.

\footnotetext{
${ }^{1}$ Médicos y cirujanos. Universidad del Cauca.

${ }^{2}$ Médica y cirujana. Universidad Libre. Santiago de Cali- Colombia.

${ }^{3}$ Residente Especialización en Ginecología y Obstetricia. Universidad del Cauca.

${ }^{4}$ Profesor asistente. Departamento Ginecología y Obstetricia. Universidad del Cauca.

* Correspondencia: John Edison Ijají Piamba. Correo electrónico: edisonijaji@outlook.com
} 


\section{Introducción}

El neumomediastino espontáneo, también conocido como Síndrome de Hamman, se diagnostica en uno de 7.000-44.000 ingresos hospitalarios y suele ocurrir en pacientes jóvenes sin comorbilidad aparente ${ }^{(1)}$. El síndrome corresponde a la presencia de aire en el mediastino, la cual no guarda relación con el trauma de tórax, los procedimientos traqueobronquiales o esofágicos, la ventilación mecánica, el cateterismo cardíaco, la cirugía torácica; o condiciones análogas $^{(2,3)}$.

Clínicamente se caracteriza principalmente por dolor torácico $(63 \%$ de los casos), disnea (44\%) y enfisema subcutáneo $(45 \%)^{(1)}$, aunque pueden aparecer: dolor del cervical, ingurgitación venosa, disfagia, disfonía y/o cambios en el tono de la voz, tos, hemoptisis, palpitaciones, polipnea, cianosis, pulso paradójico, ansiedad, enfisema retroperitoneal ${ }^{(4,7)}$.

El diagnóstico se realiza por sospecha clínica y se confirma mediante la radiografía de tórax ${ }^{(8)}$. La sensibilidad de la RX de tórax alcanza entre 91-95\% de sensibilidad para el diagnóstico, pero la especificidad podría ser deficiente ${ }^{(7)}$. La tomografía es útil cuando la radiografía de tórax resulta insuficiente o se sospechan otros diagnósticos alternativos ${ }^{(9)}$ que pudiesen comprometer la vida de la madre y/o requirieran un tratamiento urgente como tromboembolismo pulmonar, embolismo de líquido amniótico, toxicidad por anestésicos locales, mediastinitis y neumotórax a tensión, entre otras ${ }^{(10)}$. Estudios adicionales, incluyen la electrocardiografía de 12 derivaciones, la cual puede evidenciar disminución de voltaje, inversión de ondas T y cambios inespecíficos del segmento $\mathrm{ST}^{(11)}$. El tratamiento generalmente consiste en medidas de soporte basadas en la administración de oxígeno, analgesia y ansiolisis ${ }^{(7)}$. El objetivo del presente artículo es describir un caso de neumomediastino asociado al trabajo de parto.

\section{Descripción del Caso Clínico}

Presentamos el caso de una paciente de 20 años de edad, perteneciente a una etnia indígena, con gestación de 40 semanas y sin antecedentes médico-quirúrgicos relevantes. Ingresa a un Hospital de III nivel de atención de la Ciudad de Popayán, Cauca, Colombia; remitida desde nivel I de atención para cuidados postparto, ocurrido en el medio extrahospitalario.

El trabajo de parto inicia 24 horas antes del ingreso al hospital de I nivel y es vigilado por matrona en área rural. Es importante mencionar que, dentro de las costumbres de su etnia, muchos de los partos tienen lugar en el domicilio, donde son atendidos por parteras entrenadas por tradición dentro del resguardo, pero sin educación formal ginecobstétrica. De esta manera, no se pueden asegurar condiciones adecuadas de salubridad, así como tampoco se logran detectar tempranamente las alteraciones del trabajo de parto. El nacimiento y alumbramiento ocurren durante traslado en ambulancia a primer nivel de atención, solicitado luego de un trabajo de parto prolongado e infructuoso.

Al ingreso al nivel III de atención, la paciente presenta dolor torácico retroesternal de intensidad 4/10 en la escala numérica del dolor y disnea, a pesar del aporte de oxígeno suplementario. $\mathrm{Al}$ examen físico se encuentran los siguientes signos vitales: tensión arterial: 108/66 mmHg, frecuencia respiratoria: $30 / \mathrm{min}$, temperatura: $37^{\circ} \mathrm{C}$, saturación periférica de oxígeno: $96 \%$, con fracción inspirada de oxígeno (FiO2) ambiental. Además, se aprecia inyección conjuntival; edema de párpados, cara y cuello; y crépitos subcutáneos en región anterior del tórax. La auscultación cardiopulmonar es normal. El abdomen se palpa blando, doloroso en su porción inferior, con presencia de útero a nivel umbilical. Se documenta desgarro cervico-vaginal grado II, suturado en primer nivel de atención, restos placentarios a la especuloscopia, con sangrado escaso y loquios fétidos. Los resultados de imagen y laboratorio documentados al momento del ingreso a la institución se describen a continuación. Cuadro hemático. Leucocitos: $17850 / \mathrm{mm}^{3}$, neutrófilos: $14780 / \mathrm{mm}^{3}$, linfocitos: $3020 / \mathrm{mm}^{3}$, hemoglobina: $9.8 \mathrm{~g} / \mathrm{dl}$, hematocrito: $28.3 \%$, recuento de plaquetas: $229.000 / \mathrm{mm}^{3}$. Gases arteriales: PH: 7.45, PCO2: 23.1, PO2: 86.3, HCO3-: 15.8, BE: -6.7, PaO2/FIO2: 411. Los tiempos de coagulación, el fibrinógeno, así como las pruebas de función hepática y renal se reportan como normales. Rayos X de tórax: enfisema subcutáneo en los tejidos blandos de ambos hemitorax y regiones laterales del cuello, silueta cardiomediastinica no valorable por pobre esfuerzo inspiratorio, sin evidencia de consolidación, los hilios son pulmonares normales y los recesos costofrénicos se observan libres (figura 1).

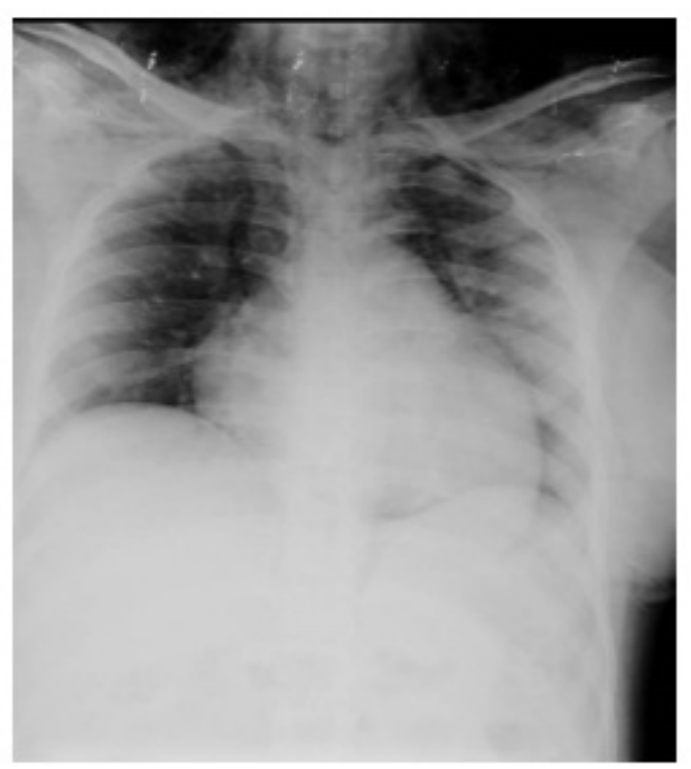

Figura 1. Rayos $\mathrm{X}$ de tórax, donde se observa enfisema subcutáneo en tejidos blandos de ambos hemitorax $\mathrm{y}$ regiones laterales del cuello. Fuente: Correspondencia de los autores. 
Con base en resultados de laboratorio, se decide inicio de terapia antibiótica con clindamicina $600 \mathrm{mg}$ EV cada 6 horas más gentamicina $160 \mathrm{mg}$ EV cada 24 horas; además de soporte de oxígeno, analgesia con diclofenaco $75 \mathrm{mg} \mathrm{EV}$ cada 8 horas y terapia hídrica con solución salina al $0.9 \%$.

Además, se realiza tomografía axial computarizada (TAC) de tórax, la cual reporta pequeñas cámaras de neumotórax bilateral, extenso neumomediastino y neumopericardio bilateral; así como severo enfisema subcutáneo facial cervical y torácico (figura 2). Con los resultados anteriores, se define mantener manejo expectante con apoyo de oxígeno con FIO2 igual al $50 \%$, analgesia ya instaurada y promoción de la movilización temprana.

Tras cinco días de estancia hospitalaria, se considera la buena evolución de la paciente y ante la ausencia de dolor y signos de dificultad respiratoria, se decide egreso con orden de terapia respiratoria ambulatoria diaria por las siguientes dos semanas y luego cada tercer día hasta completar un mes de terapia. Se desconoce evolución ambulatoria de la condición de la paciente.

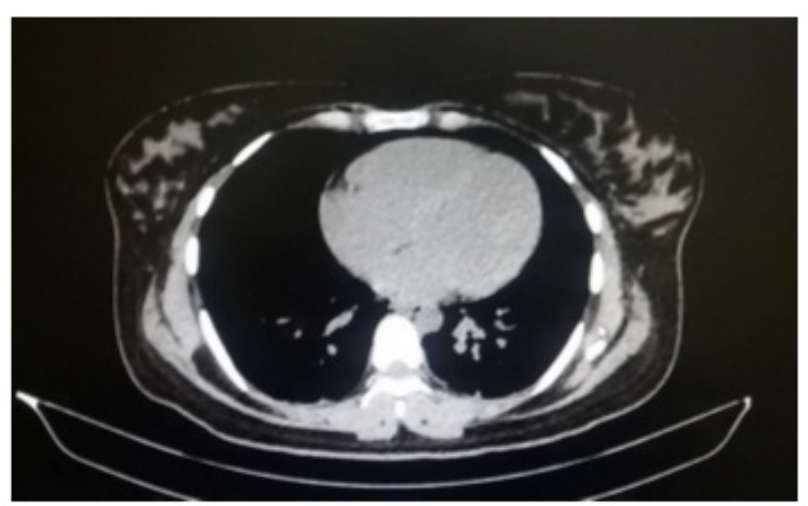

Figura 2. Corte de TAC de tórax, donde se observa neumotórax, neumomediastino y neumopericardio. Fuente: Correspondencia de los autores.

\section{Discusión}

El neumomediastino es una complicación rara dentro de la práctica obstétrica, con una incidencia calculada de uno por cada 2000 -100.000 partos y aproximadamente 200 casos reportados en todo el mundo ${ }^{(4,9,12)}$. Este generalmente se desarrolla en la segunda fase del trabajo de parto, en mujeres primigrávidas y sin comorbilidades $^{(13)}$. En las gestantes, el neumomediastino está relacionado con la maniobra de Valsalva, generada por la tos, los vómitos o el pujo durante el parto ${ }^{(4)}$. La maniobra de Valsalva incrementa la presión intratorácica; especialmente, durante la segunda etapa del trabajo cuando la mujer puja activamente, por lo que los síntomas pueden debutar en el período posparto ${ }^{(4,14)}$. Así las cosas, a pesar de su diagnóstico en el puerperio inmediato, se considera que el presente corresponde a un caso de neumomediastino con etiología en la labor de parto.

La observación clínica y estudios de extensión como la TAC de tórax, permiten descartar en su mayoría trastornos importantes. En el presente reporte de caso se acudió a esta y al fibrinógeno, además de la vigilancia clínica, para desestimar otras etiologías diagnósticas compatibles con el cuadro clínico. El neumotórax simple, el cual aparece entre 6-32\% de los casos de neumomediastino debido al trabajo de parto, fue la complicación observada en el presente caso.

En la materna, el manejo del neumomediastino asociado al trabajo de parto es igualmente conservador y está basado en la provisión de ansiolisis, analgesia y oxigenoterapia. En general, el pronóstico es bueno, con una recurrencia infrecuente en gestaciones posteriores $^{(15)}$. En el presente caso, la analgesia se brindó con diclofenaco, el cual resultó suficiente para el control del dolor. No hubo necesidad de instaurar ansiolisis, por tanto la paciente la paciente no mostró un nivel de ansiedad susceptible de manejo farmacológico. Finalmente, la administración de oxígeno constituyó el pilar del manejo expectante, el cual duró cinco días, tras los cuales fue posible el egreso hospitalario.

\section{Conclusión}

El neumomediastino es entidad de presentación excepcional durante el trabajo de parto. El diagnóstico se realiza mediante la clínica y se confirma mediante técnicas de imagen, en especial la radiografía de tórax; sin embargo, deben descartarse patologías que cursan con clínica similar y que pueden llegar a comprometer la vida de la madre. El tratamiento usualmente es expectante y se basa fundamentalmente en el aporte de oxígeno, medidas analgésicas y manejo de la ansiedad.

\section{Financiación}

Ninguna declarada por los autores.

\section{Conflictos de interés}

Ninguno declarado por los autores.

\section{Consideraciones éticas}

Los estudios de imagen fueron reproducidos bajo el consentimiento informado escrito de la paciente.

\section{Referencias}

1. Álvarez C, Jadue A, Rojas RF, Cerda C, Ramírez M, Cornejo C. Neumomediastino espontáneo (síndrome de Hamman): Una enfermedad benigna mal diagnosticada. Rev. méd. 2009:137(8):1045-50. Doi: http://dx.doi.org/10.4067/S0034-98872009000800007 
2. Silva F, Barros D, Raddatz A. Neumomediastino espontáneo (Síndrome de Hamman), una entidad poco frecuente no siempre reconocida. Rev Chil Cir.2013; 65(5):442-7. Doi: http://dx.doi.org/10.4067/S0718-40262013000500013

3. Coelho LG, Tavares de Figueiredo E, Machado Haesbaert C. Pneumomediastino espontâneo, síndrome de Hamman, relato de caso. Medicina (Ribeirão Preto. Online) 2016;49(6):574-7. Doi: https://doi.org/10.11606/issn.2176-7262.v49i6p574-5 $\underline{77}$

4. Berdai M, Benlamkadem S, Labib S, Harandou M. Spontaneous Pneumomediastinum in Labor. Case Rep Obstet Gynecol. 2017; 2017: 6235076. Article ID 6235076, 3 pages. Doi: https://doi.org/10.1155/2017/6235076

5. Kandiah S, Iswariah H, Elgey S. Postpartum Pneumomediastinum and Subcutaneous Emphysema: Two Case Reports. Case Rep Obstet Gynecol. 2013; 2013: 735154. Doi: http://dx.doi.org/10.1155/2013/735154

6. Campillo-Soto A, Coll-Salinas V, Soria-Aledo A, Blanco-Barrio A, Flores-Pastor M, Candel J, et al. Neumomediastino espontáneo: estudio descriptivo de nuestra experiencia basada en 36 casos. Arch Bronconeumol. 2005;41(9):528-31. https://doi.org/10.1157/13078656

7. Beynon F, Mearns S. Spontaneous pneumomediastinum following normal labour. BMJ Case Rep. 2011; 20:2011. pii: bcr0720114556. Doi: http://dx.doi.org/10.1136/bcr.07.2011.4556

8. Campbell S, Vargas SA, Gómez JM, Escobar AE,
Muñoz JK. Síndrome de Hamman. Acta Med Colomb. 2016;41(3):206-10.

9. Çintesun E, <ncesu Çintesun FN. Spontaneous pneumomediastinum and pneumothorax developing after vaginal delivery. Perinatal J. 2016;24(3):174-7.

10. Mondello B, Pavia R, Ruggeri P, Barone M, Barresi $\mathrm{P}$, Monaco $\mathrm{M}$. Spontaneous pneumomediastinum: experience in 18 adult patients. Lung. 2007;185(1):914.

11. Krause H, Portmann C. Pneumomediastinum following vaginal delivery. ANZJOG. 2000;40(1):106-7. Doi:

https://doi.org/10.1111/j.1479-828X.2000.tb03181.x

12. Khurram D, Patel B, Farra W. Hamman's Syndrome: A Rare Cause of Chest Pain in a Postpartum Patient. Case Rep Pulmonol. 2015;2015:201051.Doi: http://dx.doi.org/10.1155/2015/201051

13. Kouki S, Fares AA. Postpartum spontaneous pneumomediastinum 'Hamman's syndrome'. BMJ Case Rep. 2013;2013. pii: bcr2013010354.

http://dx.doi.org/10.1136/bcr-2013-010354

14. Spronk PE, Landheer W, Wessels F, Kardux JJ, Huisman M. Hamman's síndrome. Neth J Crit Care. $2011 ; 15(1): 30-1$.

15. Yadav Y, Ramesh L, Davies JA, Nawaz $\mathrm{H}$, Wheeler R. Gross spontaneous pneumomediastinum (Hamman's syndrome) in a labouring patient. J Obstet Gynaecol. 2008;28(6):651-2. Doi: https://doi.org/10.1080/01443610802378058 